\title{
Performance of Eucalyptus and OpenStack Clouds on FutureGrid
}

\author{
Sharath S \\ Scholar, CSE Department, \\ East Point College of Engineering and Technology, \\ Bengaluru 560068, Karnataka, India.
}

\author{
Anirban Basu \\ Professor and Head, CSE Department, \\ East Point College of Engineering and Technology, \\ Bengaluru 560068, Karnataka, India.
}

\begin{abstract}
With expanding demand for Cloud computing, numerous Cloud providers are offering services. Benchmarking is extremely important to compare their performance. In this paper, we study the performance of two IaaS clouds Eucalyptus 3 and OpenStack (version Essex) implemented on FutureGrid [1,2], a test bed that allows researchers to conduct experiments to understand the behavior and utility of cloud computing services. Phoronix Test Suite [3] is utilised as a benchmark program to conduct diverse performance measurements on both the clouds. Besides the programs in Phoronix Test suite, time to launch instances, time to register images using scripts were also matched. The results are discussed in this paper.
\end{abstract}

\section{General Terms}

Virtual Machines, Benchmark, Cloud computing.

\section{Keywords}

FutureGrid, Eucalyptus, OpenStack, Phoronix Test Suite, Image registration time.

\section{INTRODUCTION}

A Cloud computing platform has a large pool of resources which can be accessed by users on demand. The resources shared by a number of users are allocated for better production and for optimum resource utilization.Users submit their jobs (or demands for resources such as CPU, RAM, disk, application, etc.) over the network. Depending upon the service requirement a Cloud computing platform configures its resources dynamically. Cloud computing platforms offer three types services which are:

- Infrastructure-as-a-Service (IaaS)

- $\quad$ Platform-as-a-Service (PaaS)

- $\quad$ Software-as-a-Service (SaaS).

Examples of IaaS cloud include Amazon EC2, IBM Cloud. Microsoft Azure and Google AppEngine are examples of PaaS cloud. Examples of SaaS include Gmail, Google Docs.

In this paper we focus on the performance of open source IaaS type of cloud computing platforms. Two popular open source IaaS Cloud Computing environments are: Eucalyptus [4, 5] and OpenStack [6]. These environments are widely established in private and public clouds and FutureGrid deploys both of these. FutureGrid encompasses a geographically accessible set of heterogeneous computing systems, a data administration system, and a library of software images. FutureGrid runs on a network accessible to users in several countries allowing researchers to conduct experiments.
A significant feature of cloud computing is virtualization of physical resources which enables the execution of multiple jobs on the same, shared physical environment by creating Virtual Machines (VMs). This make a cloud based service economical to both cloud service providers and cloud users. Cloud service providers can reduce their costs of service delivery by resource consolidation through virtualization.

Benchmarking plays a critical role in evaluating the performance of systems that are ready for operation. With the increasing use of cloud computing, more and more users are seeking answers to questions on which type of cloud they should use for services at an economical cost.

While work is going on designing appropriate benchmarks, so far Phoronix Test Suite [3] is broadly used. In this paper we discuss the results obtained by running the Phoronix Test suite on Eucalyptus and OpenStack on FutureGrid. For measuring the time for registration and launch of VMs on the clouds, scripts had to be written.

\section{RELATED WORK}

Benchmarking is necessary because of wide-spread adoption of cloud across world. Alexander Lenk, Michael Menzel, Johannes Lipsky, Stefan Tai and Philipp Offermann have designed performance measuring procedure for Infrastructureas-a-Service and evaluated Amazon EC2 platform, Flexiscale platform and Rackspace platform to validate its utility [7]. Taxonomy to standardize performance evaluation of commercial Cloud services is provided in [8] and distinct characteristics that are to be considered while evaluating performance of Cloud are discussed. In [9] comparison of four commercial cloud providers Amazon EC2, Windows Azure, Google AppEngine and Rackspace CloudServers has been done including several components of computing, network, database and storage. The startup time of cloud VMs across Amazon EC2, Windows Azure and Rackspace and different factors that affect startup time are studied in [10].

\section{CLOUD FRAMEWORKS USED}

\subsection{FutureGrid}

FutureGrid[2] is a distributed, high-performance test-bed that permits scientists to collaboratively work and design innovative approaches to parallel, grid, and cloud computing. The FutureGrid project is funded by the National Science Foundation (NSF) and is led by Indiana University, University of Chicago, University of Florida, San Diego Supercomputing Center, Texas Advanced Computing Center, University of Virginia, University of Tennessee, University of Southern California, Dresden, Purdue University, and Grid 5000 .

FutureGrid permits researchers to conduct experiments in computer science related to grid and cloud computing. 
FutureGrid provides IaaS and PaaS frameworks and supports three cloud services: Eucalyptus, OpenStack and Nimbus.

\subsection{Eucalyptus}

Eucalyptus is a software platform that applies IaaS type cloud computing [4, 5]. Eucalyptus presents Amazon EC2 based web service interface for cloud service. Eucalyptus on FutureGrid is comprised of five components:

- Cloud Controller is the entry point into the cloud. It interacts with other constituents and organizes underlying virtualized resources (servers, network and storage).

- Walrus [4] allows users to store persistent data as buckets and objects.

- Storage Controller provides functionality to create Elastic Block Storage (EBS) volumes. EBS volumes store continual data of virtual machines.

- $\quad$ Node Controller controls virtual machine activities including execution, examination and termination of VM instances.

\subsection{OpenStack}

OpenStack is a collection of open source components to deliver public and private clouds that supply IaaS [6]. OpenStack on FutureGrid includes following constituents:

- Nova is designed to provision and manage large networks of virtual machines.

- Swift provides object storage to store or retrieve files.

- Glance is responsible for discovery, registration and delivers services for virtual disk images.

\section{PHORONIX TEST SUITE}

The Phoronix Test Suite [3, 4] is the most comprehensive benchmarking suite of programs available to effectively carry out performance evaluation and is also very simple to use.

The Phoronix Test Suite is utilised for determining computer's performance for internal quality assurance purposes, and hardware validation. It runs on platforms ranging from smartphones and personal computers to multicore workstations and cloud computing infrastructures. It is developed for Linux, OpenSolaris, Apple Mac OS X, Microsoft Windows, and BSD operating systems.

\section{IMPLEMENTATION AND RESULTS}

We created new FutureGrid portal account and used IaaS services to create necessary virtual machines. Phoronix Test Suite software was installed in different types of instances of both clouds. Benchmark test in this suite were run to find performance of instances. Shell scripts were written for registering images and computing the time taken for registering. Launch time of instances furthermore discovered using shell scripts

Phoronix Test Suite is a collection of test profiles with a granted set of test options. Phoronix Test Suite is used to find performance of virtual machines related to aiostress, ramspeed, and compress-gzip and so on.

Openstack in FutureGrid has following types of Virtual Machines (see Table 1).
Table 1: OpenStack VM types in FutureGrid

\begin{tabular}{|l|l|l|l|}
\hline VM type & $\begin{array}{l}\text { RAM } \\
\text { (MB) }\end{array}$ & DISK (GB) & VCPUs \\
\hline m1.tiny & 512 & 0 & 1 \\
\hline m1.small & 2048 & 10 & 1 \\
\hline m1.medium & 4096 & 10 & 2 \\
\hline m1.large & 8192 & 20 & 4 \\
\hline m1.xlarge & 16384 & 40 & 8 \\
\hline
\end{tabular}

As m1.tiny is rarely used this case is not considered. When m1.small type virtual machines are created 10 GB disk space and one virtual CPU is allocated.

Eucalyptus in FutureGrid has following types of Virtual Machines (see Table 2).

Table 2: Eucalyptus VM types in FutureGrid

\begin{tabular}{|l|l|l|l|}
\hline VM type & $\begin{array}{l}\text { RAM } \\
\text { (MB) }\end{array}$ & DISK (GB) & VCPUs \\
\hline m1.small & 512 & 5 & 1 \\
\hline c1.medium & 2048 & 7 & 1 \\
\hline m1.large & 5120 & 10 & 2 \\
\hline m1.xlarge & 6000 & 15 & 2 \\
\hline c1.xlarge & 9216 & 20 & 4 \\
\hline
\end{tabular}

We represent OpenStack instance types with prefix "s" and Eucalyptus instances with prefix "e". Results obtained are presented here.

\subsection{Disk Performance}

\subsubsection{AIO-Stress:}

This is asynchronous I/O benchmark using 2048 MB test file and $64 \mathrm{~KB}$ record size. OpenStack virtual machines have lower I/O stress compared to Eucalyptus virtual machines. As shown in Figure 1, I/O stress increases with size. Eucalyptus instances have higher I/O stress.

\subsection{Processor Performance}

\subsubsection{Build Linux Kernel:}

This test measures the time taken to compile and build Linux kernel. As shown in Figure 2 even though smaller instance types of Eucalyptus take larger time compared to OpenStack, larger instances take less time.

5.2.2 Byte:

\subsubsection{Dhrystone}

This standard is used to measure and compare the performance of Virtual Machines. The test focuses on string handling and is heavily leveraged by hardware and software design, compiler and linker options, code optimization, cache memory, wait states, and integer data types[. (See figure 3) 


\subsubsection{Floating-Point Arithmetic:}

This test measures the speed and effectiveness of floatingpoint operations. A wide range of $\mathrm{C}$ functions including sin, cos, sqrt, exp, and log are used as well as integer and floatingpoint math operations, array accesses, conditional branches, and procedure calls (See figure 4).

\subsubsection{Gzip Compression:}

This test measures the time needed to compress a file using Gzip compression. Eucalyptus instance types almost take same amount of time around 26 seconds. All OpenStack instance types take less time compared to Eucalyptus except m1.small because of high processor speed (See figure5).

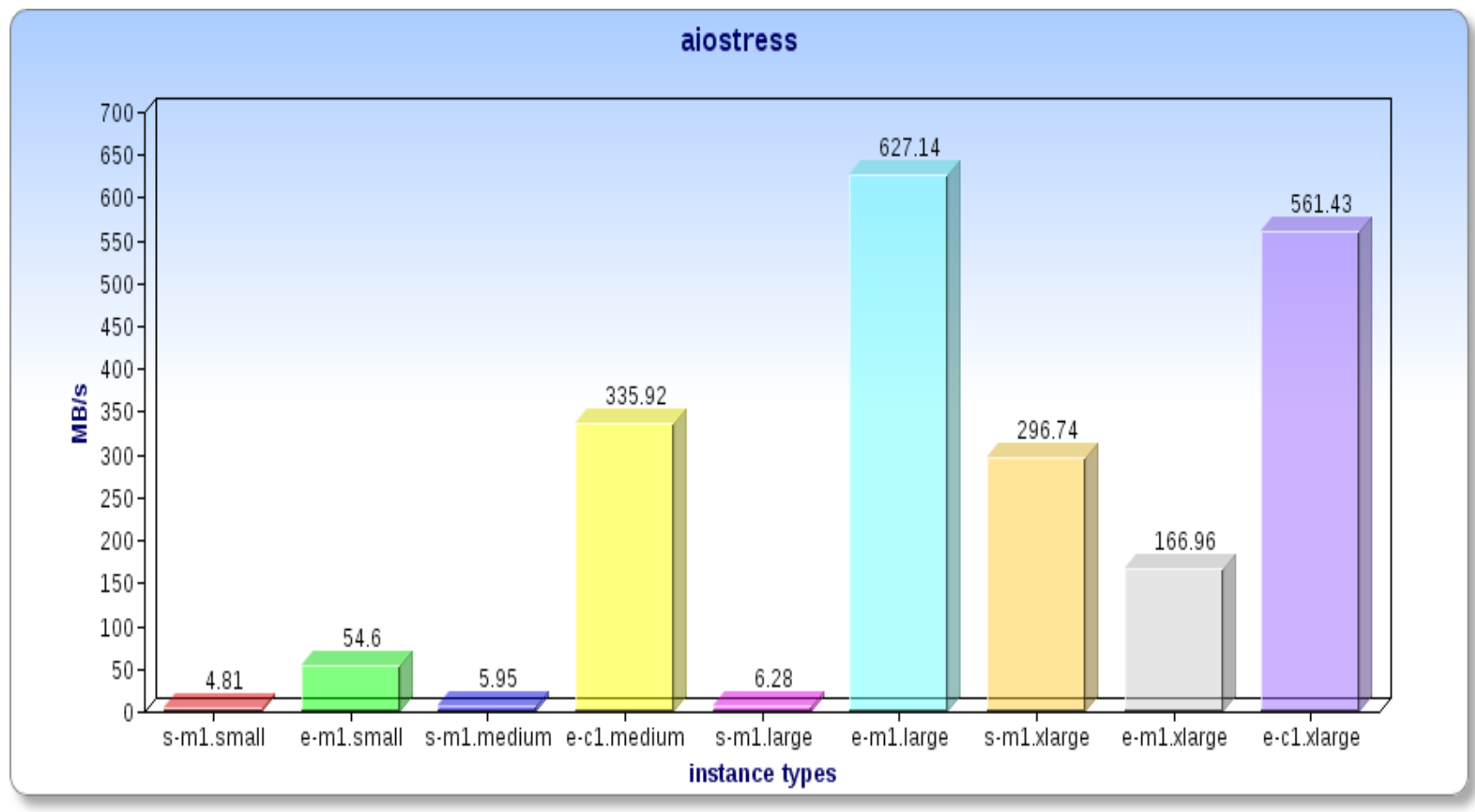

Figure 1: AIO-Stress

\section{build linux kernel}

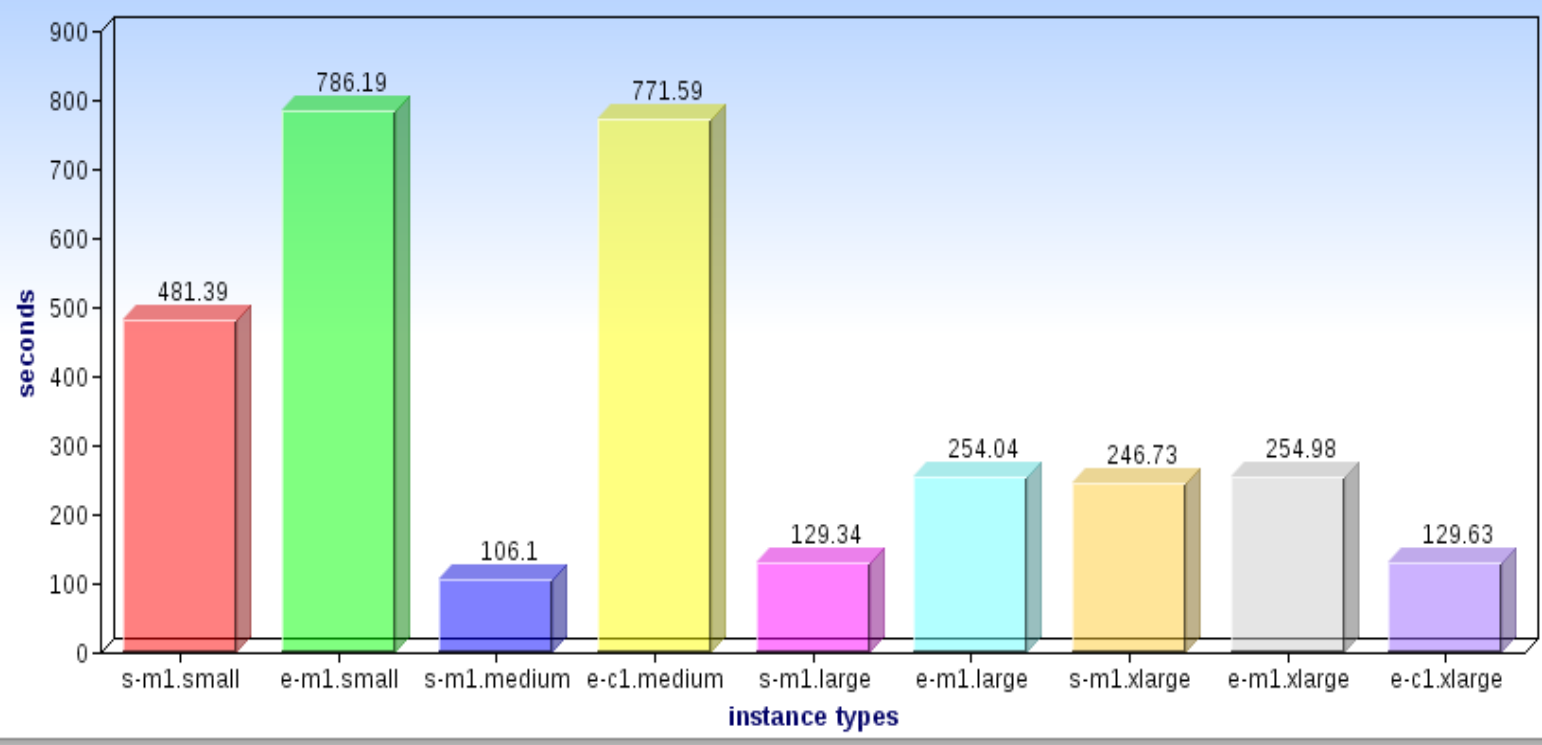

Figure 2: Build Linux Kernel 


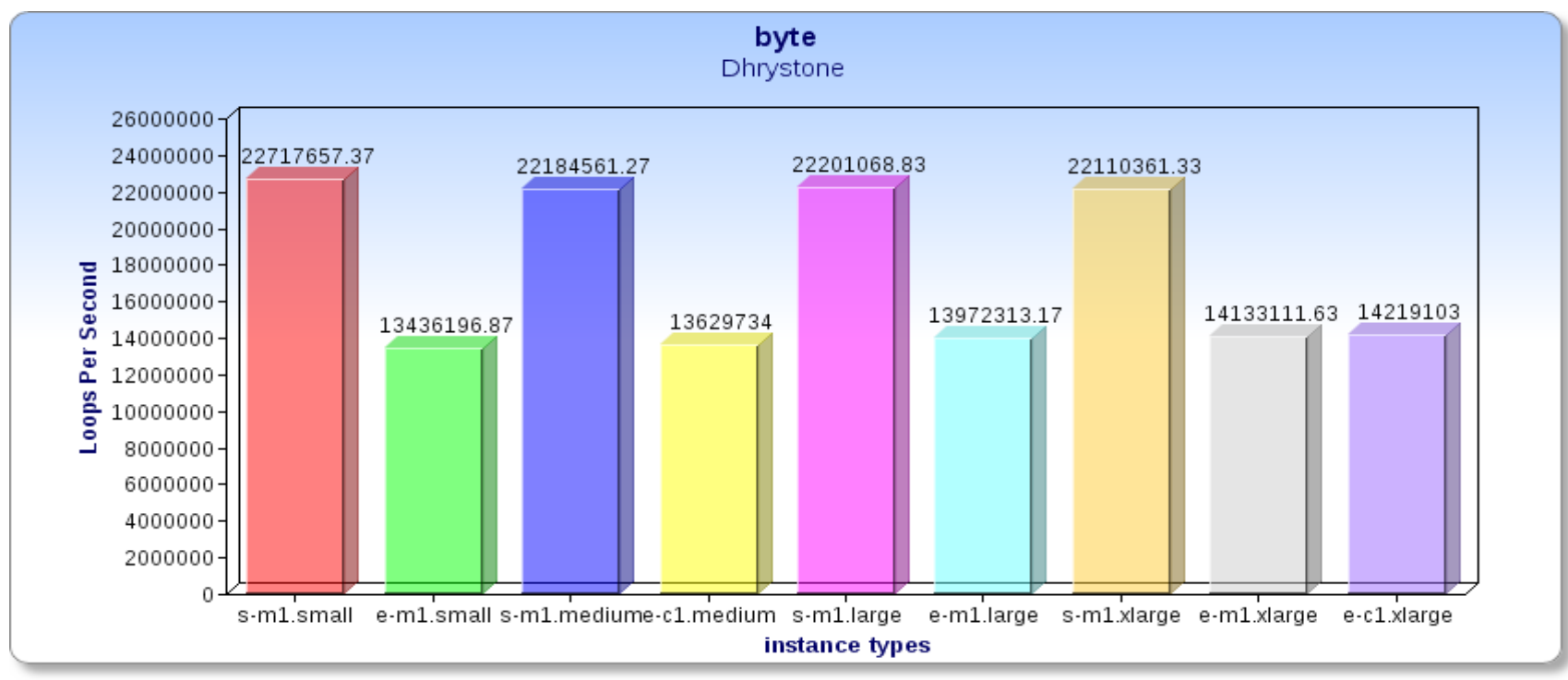

Figure 3: Dhrystone

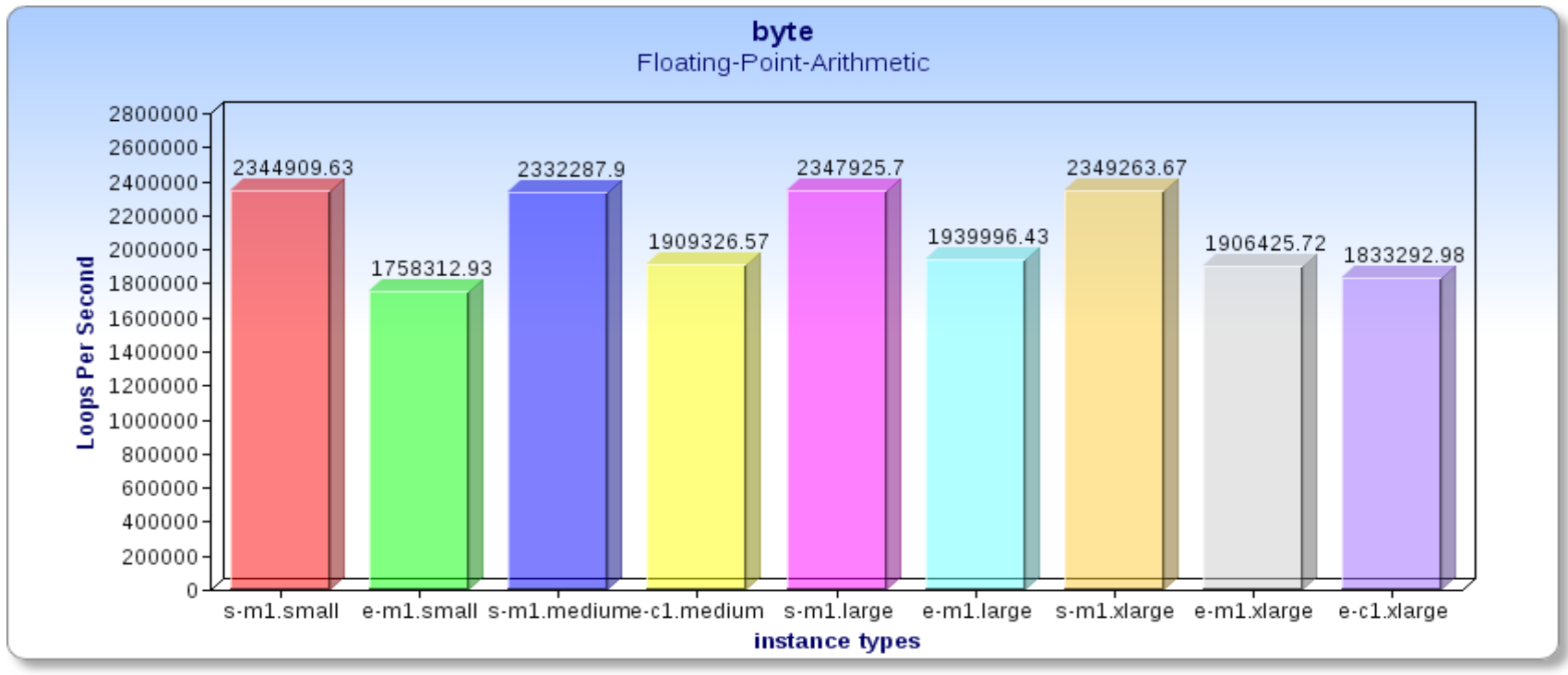

Figure 4: Floating-Point Arithmetic

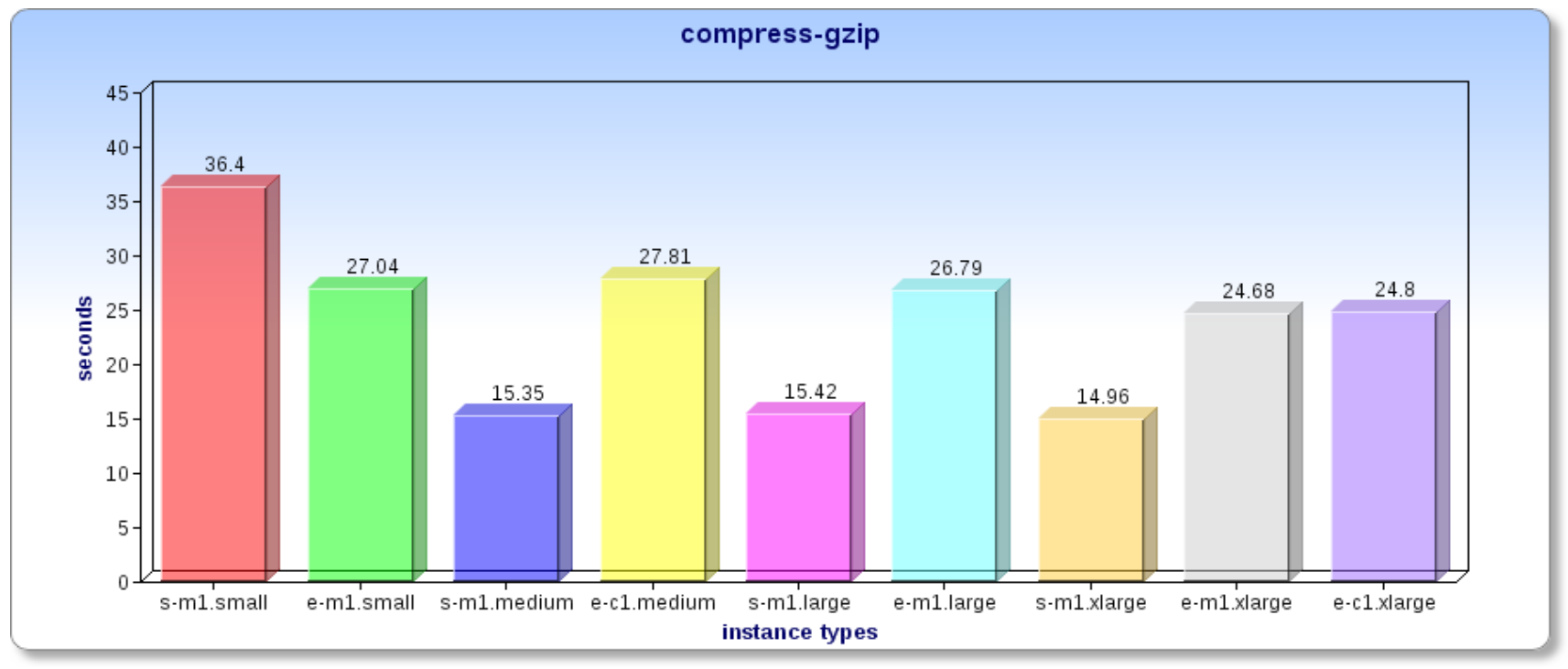

Figure 5: Gzip Compression 


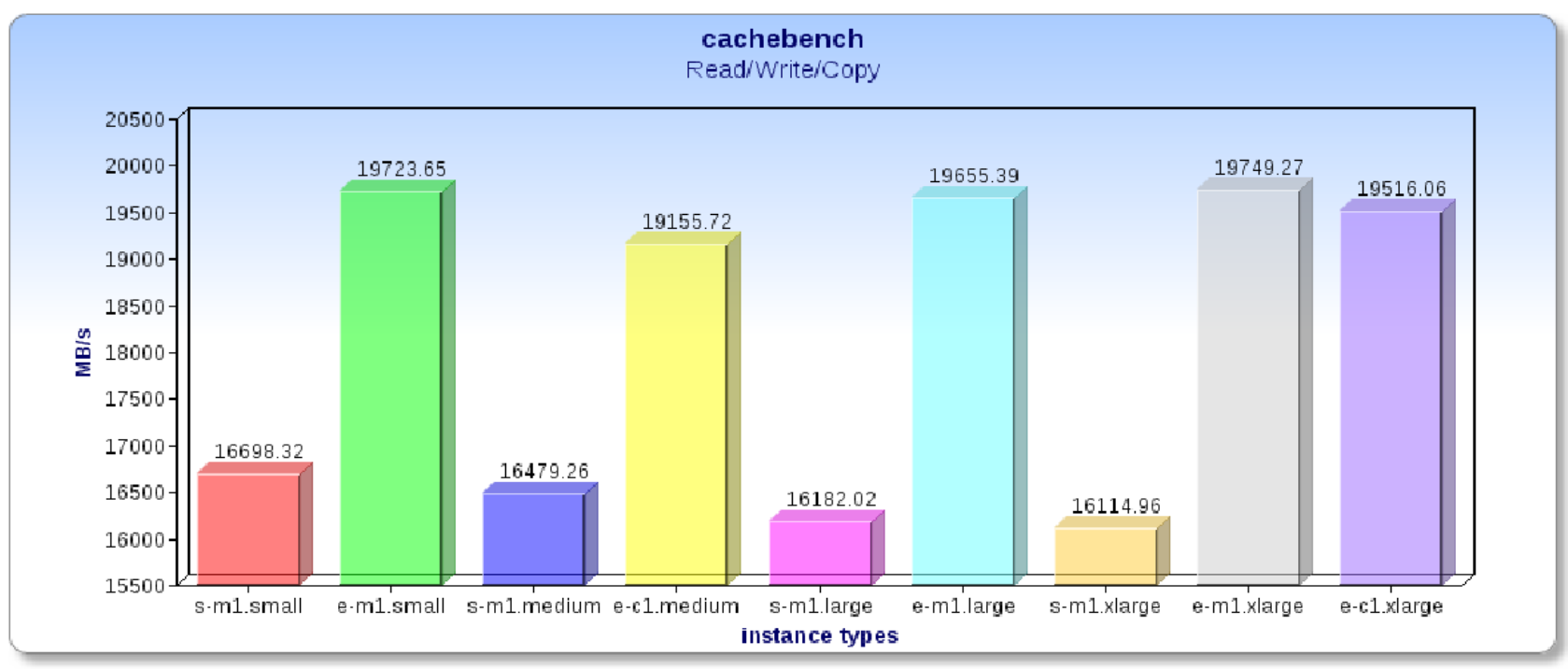

Figure 6: CacheBench

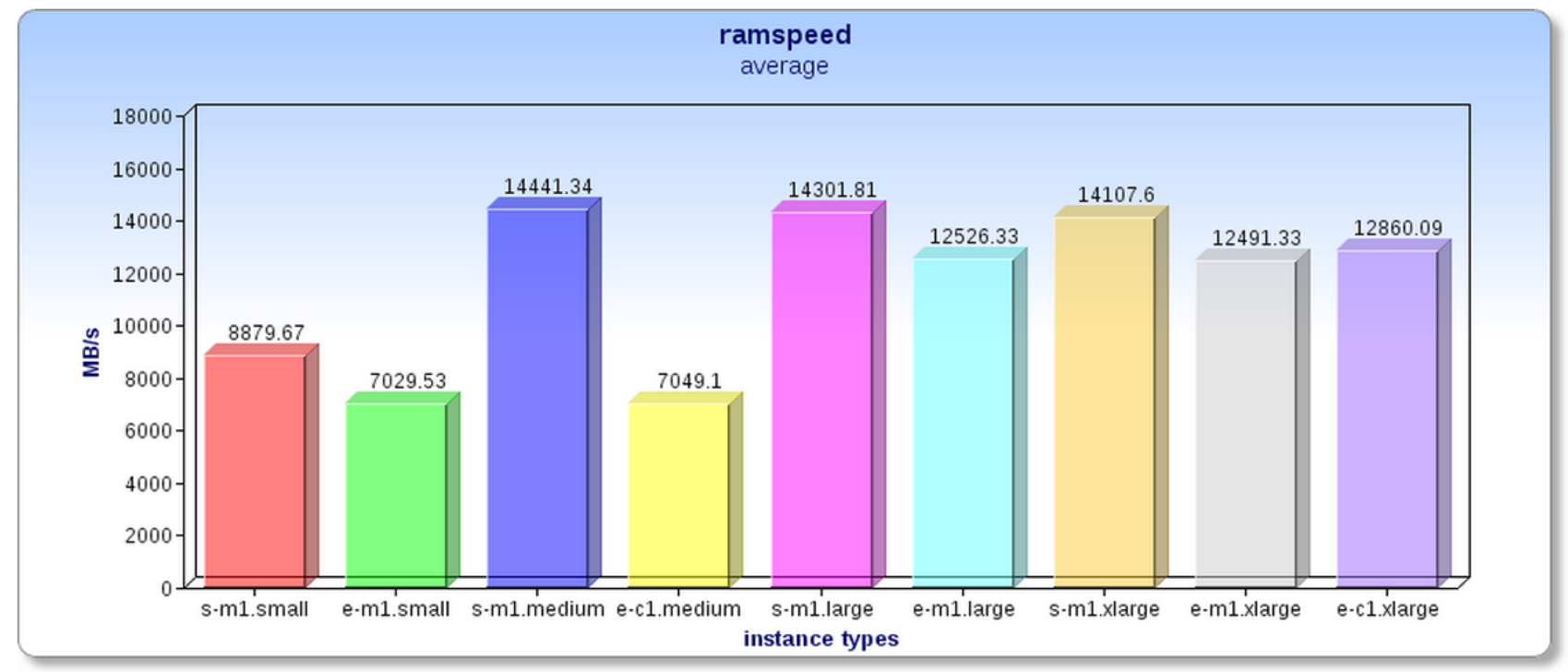

Figure 7: RAMspeed SMP (System Memory Performance)

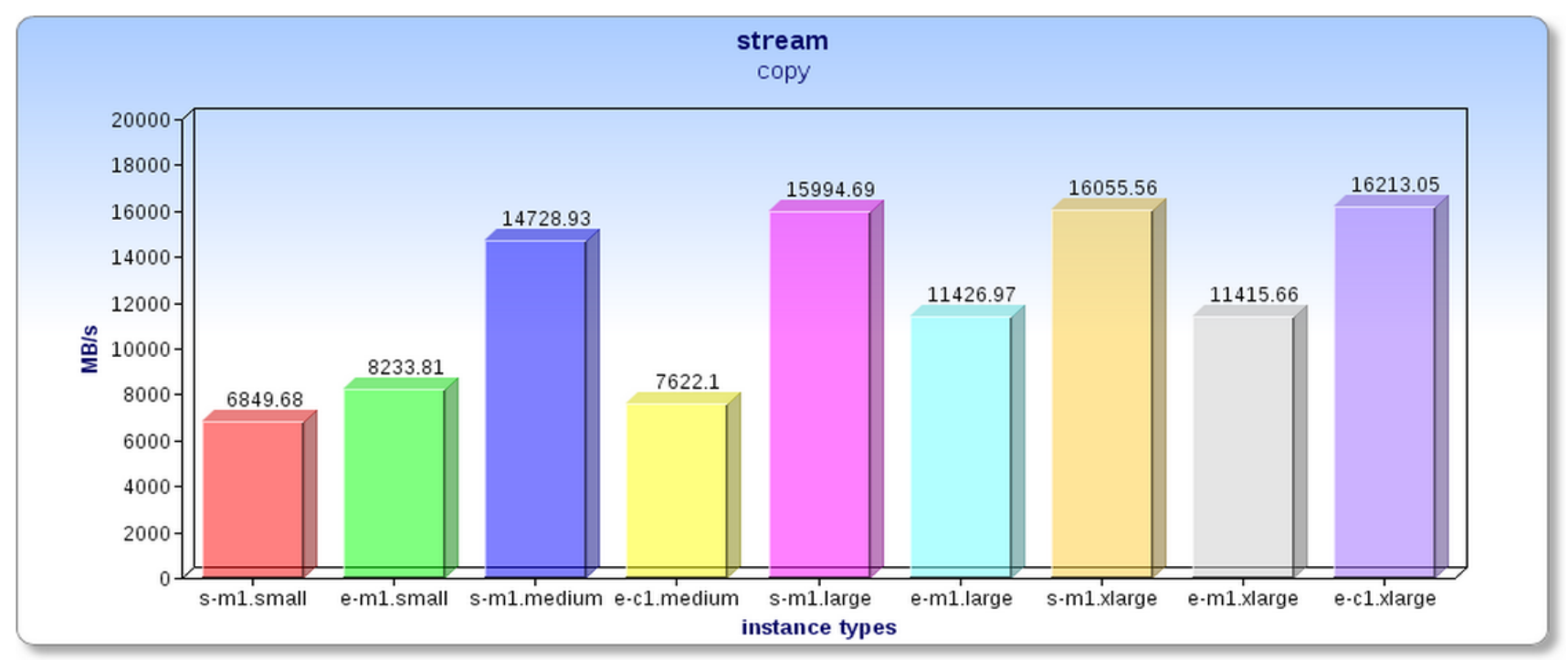

Figure 8: Stream 


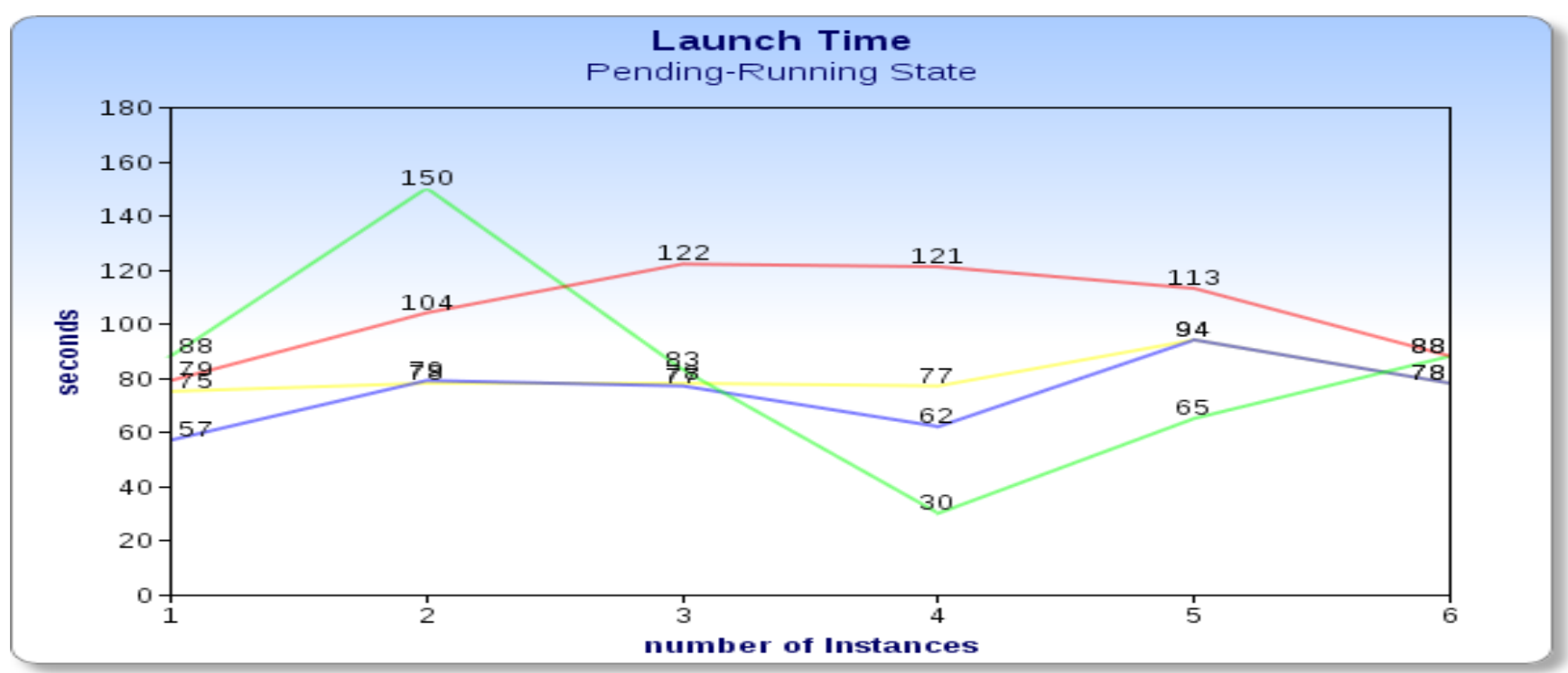

e-c1.medium s-m1.small s-m1.medium e-m1.large

Figure 9: Virtual machines launch time

\subsection{Memory Performance}

\subsubsection{CacheBench:}

CacheBench is a standard designed to assess the performance of the memory hierarchy of computer systems. This performs recurring access to data items on varying vector lengths. Timings are taken for each vector length over a number of iterations. Computing the product of iterations and vector length presents us the total amount of data accessed in bytes. This total is then divided by the total time to compute bandwidth. Results in Figure 6 show that even though OpenStack processors are faster Eucalyptus instances perform better in this test.

\subsubsection{RAMspeed:}

This benchmark tests the system memory (RAM) performance. RAMspeed tests how fast are cache and memory subsystems by assigning certain memory space and start either writing to or reading from it using continuous blocks sized in power of 2 from $1 \mathrm{~Kb}$ up to the array boundary. They function with sequential data streams passed through ALU, FPU units respectively. OpenStack virtual machines are found to have more bandwidth compared to Eucalyptus virtual machines (See figure 7).

\subsubsection{Stream:}

The stream benchmark is a simple synthetic benchmark program that measures sustainable memory bandwidth and the corresponding computation rate for simple vector kernels. The standard is specifically designed to work with datasets much larger than the available cache on any allocated system. Eucalyptus c1.xlarge performs better than all other kinds, but overall OpenStack instances have good bandwidth (See figure 8).

\subsection{Virtual machines launch time}

Shell scripts were written to run virtual machines sequentially. Time taken by virtual machines to change from pending to running state is computed which gives the time taken to acquire resources (start time or launch time). Eucalyptus virtual machines take less time contrasted to OpenStack virtual machines (See figure 9).

\subsection{Image registration time}

This includes time taken to bundle, upload and register an image. The supported kernel and ramdisk images available in FutureGrid should be chosen for registering custom images. Two images of $1.5 \mathrm{~GB}$ and $3 \mathrm{~GB}$ are registered to Eucalyptus and OpenStack (See figure 10).

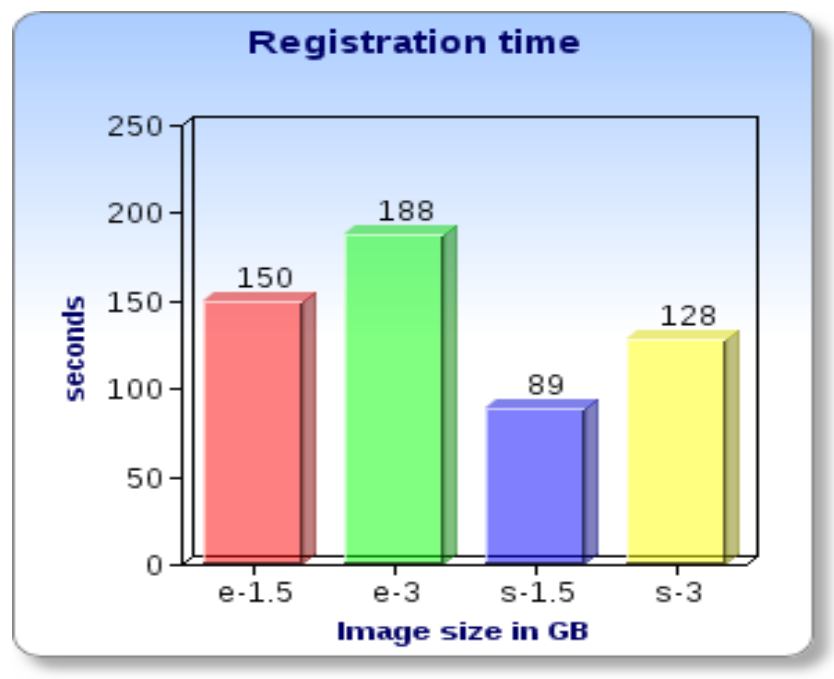

Figure 10: Image registration time

\section{CONCLUSION}

Phoronix Test Suite was used to benchmark both Eucalyptus and OpenStack Cloud Computing platforms. Outcomes show that Openstack instances have lower IO/stress and higher processing speed. Eucalyptus instances have higher cache and RAM memory bandwidths. Eucalyptus is apt for cache/memory intensive applications and OpenStack for CPU intensive applications. Using shell-scripts we conclude that Eucalyptus instances take slightly lesser time to start running compared to OpenStack types. OpenStack registers images more quickly than Eucalyptus. Thus we conclude in 
FutureGrid Openstack Essex outperforms Eucalyptus 3 in most cases.

\section{REFERENCES}

[1] FutureGrid web page, https://portal.futuregrid.org/, July 2013

[2] Gregor von Laszewski, Javier Diaz, Fugang Wang, Geoffrey C. Fox, "Comparison of Multiple Cloud Frameworks", $5^{\text {th }}$ International Conference on Cloud Computing, IEEE 2012

[3] Phoronix Test Suite web page, https://www.phoronixtest-suite.com, July 2013

[4] Eucalyptus web page, http://open.eucalyptus.com/, July 2013.

[5] Daniel Nurmi, Rich Wolski, Chris Grzegorczyk, Graziano Obertelli, Sunil Soman, Lamia Youseff, Dmitrii Zagorodnov, "The Eucalyptus Open source Cloud computing System" In Proceedings of the 9th IEEE/ACM International Symposium on Cluster Computing and the Grid, CCGrid, China, 2009.
[6] OpenStack web page, http://www.openstack.org, July 2013.

[7] Alexander Lenk, Michael Menzel, Johannes Lipsky, Stefan Tai, Philipp Offermann, "What are you paying for? Performance benchmarking for Infrastructure-as-aService offerings", 4th International Conference on Cloud Computing, IEEE 2011.

[8] Zheng Li, Liam O'Brien, Rainbow Cai, He Zhang, "Towards a Taxonomy of Performance Evaluation of Commercial Cloud Services", $5^{\text {th }}$ International Conference on Cloud Computing, IEEE 2012

[9] A. Li, X. Yang, S. Kandula, and M. Zhang, "Cloudemp: comparing public cloud providers," in Proceedings of the $10^{\text {th }}$ annual conference on Internet measurement, ACM 2010 .

[10] Ming Mao, Marty Humphrey, "A Performance Study on the VM Startup Time in the Cloud", $5^{\text {th }}$ International Conference on Cloud Computing, IEEE 2012 